\title{
The scenario of two families of compact stars
}

\section{Transition from hadronic to quark matter and explosive phenomena}

\author{
Alessandro Drago and Giuseppe Pagliara \\ Dip. di Fisica e Scienze della Terra dell'Università di Ferrara and INFN Sez. di Ferrara, Via Saragat 1, I-44100 Ferrara, Italy
}

Received: date / Revised version: date

\begin{abstract}
We will follow the two-families scenario described in the accompanying paper, in which compact stars having a very small radius and masses not exceeding about $1.5 M_{\odot}$ are made of hadrons, while more massive compact stars are quark stars. In the present paper we discuss the dynamics of the transition of a hadronic star into a quark star. We will show that the transition takes place in two phases: a very rapid one, lasting a few milliseconds, during which the central region of the star converts into quark matter and the process of conversion is accelerated by the existence of strong hydrodynamical instabilities, and a second phase, lasting about ten seconds, during which the process of conversion proceeds as far as the surface of the star via production and diffusion of strangeness. We will show that these two steps play a crucial role in the phenomenological implications of the model. We will discuss the possible implications of this scenario both for long and for short Gamma Ray Bursts, using the proto-magnetar model as the reference frame of our discussion. We will show that the process of quark deconfinement can be connected to specific observed features of the GRBs. In the case of long GRBs we will discuss the possibility that quark deconfinement is at the origin of the second peak present in quite a large fraction of bursts. Also we will discuss the possibility that long GRBs can take place in binary systems without being associated with a SN explosion. Concerning short GRBs, quark deconfinement can play the crucial role in limiting their duration. Finally we will shortly revisit the possible relevance of quark deconfinement in some specific type of Supernova explosions, in particular in the case of very massive progenitors.
\end{abstract}

PACS. PACS-key describing text of that key - PACS-key describing text of that key

\section{Introduction}

In the accompanying paper (here and in the following paper 1) we have discussed the two-families scenario, in which compact stars having a mass not exceeding about $1.5 M_{\odot}$ are made of hadrons, while the most massive compact stars are entirely made of quarks, i.e. they are quark stars [1. We have also discussed the interesting mass range, located about $(1.35-1.5) M_{\odot}$, which can be populated both by hadronic and by quark stars. The latters have a significantly larger radius and a larger moment of inertia. This scenario is somehow opposite respect to the more traditional one in which quark stars are extremely compact with radii even smaller than about $10 \mathrm{~km}$.

In this second paper we discuss how the transition from a hadronic star into a quark star can take place and which are the phenomenological implications of that transition.

First, by looking at the plot of the two families already discussed in the accompanying review paper on the EoS one can recognize the possible situations in which quark matter and therefore quark stars can form. There are essentially three situations:

Send offprint requests to:
- via mass accretion or via slowing-down of a rapidly rotating pulsar, having a mass close to the critical one. This situation can for instance be realized in LMXBs, in which the neutron star accretes mass and angular momentum from the companion. Under those conditions the critical deconfinement density can be reached (maybe more easily soon after the mass accretion stops and the star starts slowing-down 2]). In these cases the temperature immediately before deconfinement is quite low and plays no role. We will link this scenario to the possibility of having long GRBs not connected with a SN explosion. See also the possible signature of the transition in the anomalous value of the eccentricity, as discussed in paper 1 ;

- soon after the SN explosion of a massive progenitor. It is possible that a delay exists between the moment the SN explodes and the moment quark deconfinement takes place: it can be due again to the gradual slowdown of the neutron star or to mass accretion due to the fall-back. This scenario can be linked to long GRBs displaying two active periods separated by a quiescent time. The possibility that the neutrino flux generated by the phase transition can help revitalizing 
a marginally failed SN explosion is not ruled out either. In this case the temperature before deconfinement can be larger, order of $20-30 \mathrm{MeV}$. Although these temperatures can facilitate quark deconfinement they still should not play a crucial role;

- after the merging of two neutron stars in a binary system. If a massive compact star forms immediately after the merging, in our scheme that star is unavoidably a quark star. This is maybe the most precise and striking signature of the two-families scenario. Since these mergers are supposed to be at the origin of short GRBs we expect to see rather clear signatures of the formation of a quark star in the features of those GRBs. Also, the emission in gravitational waves associated with the process of merging should bear the imprint of the transition from the hadronic EoS to the quark EoS (see the review paper by Bauswein et al. of this volume). In the process of merging very high temperatures are reached (up to about $50 \mathrm{MeV}$ [3] and even larger if the heat released by quark's deconfinement is taken into account) and we will see that they play quite a significant role in the phenomenology.

The most important point about the microphysics of the transition from hadronic matter to quark matter concerns under which conditions the process of deconfinement can start taking place. The very beginning of the process is the formation of a droplet of quark matter, stable at the pressure at which it forms. In the two-families scenario that we are discussing the formation of quark stars depends on the validity of the Bodmer-Witten hypothesis on the absolute stability of strange quark matter [4,5]. If strange quarks play a role in the stability of the first droplet than it is clear that the process of deconfinement cannot start unless some strangeness content already exists in the hadronic phase (we will come back to this point when discussing the possible impact of quark deconfinement on SN explosions). Statistical fluctuations of the flavor composition of a small amount of matter can facilitate the formation of an energetically favorable droplet of quark matter even if the average strangeness content is not (yet) the optimal one (more strangeness can form later if the droplet can live long enough that weak interactions can take place). On the other hand, if the hadronic phase does not contain any strangeness, either in the form of hyperons or in the form of condensed kaons, then a droplet of quark matter with a non-vanishing strangeness content cannot form on the time-scale of strong interaction, which is the one associated with the fluctuations of hadrons into deconfined quarks 6 . We therefore assume that the minimal value for the critical density corresponds to the one at which hyperons (or kaons) start forming. While this density is well defined at zero temperature, at finite temperature hyperons form at any density, although their fractional density is very small at low temperatures and low densities. The exact conditions at which the first droplet of stable strange quark matter can form at finite temperature are complicated. A first attempt in exploring that problem has been made in a few papers $[7,8,9$, but it will likely require more investigations to be completely clarified. We will not discuss in details the process of formation of the first droplet of quark matter, because it is analyzed in the papers of Lugones and Bombaci et al. of this volume.

The whole process of quark deconfinement in a stellar object can be divided in different steps:

- via quantum fluctuations (if the temperature of the system is low) or thermal fluctuations (if the temperature is large) a first droplet of quark matter forms, large enough to keep expanding;

- the droplet keeps expanding (or it merges with other droplets) till its size becomes macroscopic. This second step has, to our knowledge, never been analyzed;

- the further expansion of the macroscopic bubble of quark matter inside the hadronic star can be described by using hydrodynamical equations and it divides into two sub-steps [10]:

- a rapid burning, whose velocity is greatly augmented by hydrodynamical instabilities. It lasts only a few milliseconds and it burns the central area of the star;

- a slow burning, due to production and diffusion of strangeness, lasting some ten seconds and transforming the star into a quark star.

As we will see, the final process of burning can depend on the mass of the star to be transformed into a quark star and on its initial temperature. Two sub-cases, at least, need to be discussed: the case in which the mass of the deconfining star is about (1.4-1.5) $M_{\odot}$, the typical situation of deconfinement of a single star via mass accretion, and the case of deconfinement immediately after the merging of two compact stars in a binary system, forming a new compact object with a mass exceeding $2 M_{\odot}$. In this second case the temperature is quite larger and this will have important phenomenological implications.

\section{Burning of hadronic stars into quark stars}

Since the formulation of the Bodmer-Witten hypothesis [4,5] and its implication on the existence of compact stars entirely composed by quark matter [11,12, the process of conversion of hadronic stars into quark stars has been the subject of many theoretical investigations. At the microscopic level this process is extremely complicated because it involves the deconfinement of quarks (driven by the strong interaction) and flavor changing reactions among quarks (driven by the weak interaction). In particular the process of deconfinement is clearly the most complicated due to its non-perturbative nature. The simple kinetic theory approach proposed in Ref. [13] is still one of the most widely used: the conversion is described as a slow combustion by means of a one dimensional stationary reactiondiffusion-advection equation for the strange quarks concentration. The two key quantities in this approach are the quark diffusion coefficient $D\left(D \sim 10^{-1} \mathrm{~cm}^{2} / \mathrm{sec}\right.$ for $\mu_{q} \sim 300 \mathrm{MeV}$ and $T \sim 10 \mathrm{MeV}$ [14] $)$ and the time of conversion of down quarks into strange quarks $\tau\left(\tau \sim 10^{-9}\right.$ 
sec for $\mu_{q} \sim 300 \mathrm{MeV}$ [15]). By simple dimensional analysis (see [16]) one can obtain an estimate of the width of the combustion zone $\delta \sim \sqrt{D \tau} \sim 10^{-5} \mathrm{~cm}$ and of the burning velocity $v \sim \sqrt{D / \tau} \sim 10^{3}-10^{4} \mathrm{~cm} / \mathrm{sec}$.

Within the kinetic theory approach of [13] one does not take into account possible macroscopic collective flows and hydrodynamical instabilities driven by pressure and density gradients between the fuel and the ashes fluids. On the other hand, in the context of type Ia Supernovae, in which the nuclear burning occurs, the Rayleigh-Taylor and the Landau-Darrieus instabilities have been proven to turn the laminar combustion into a much faster turbulent combustion [17, 18. In principle one should couple the equations of hydrodynamics (i.e. the equations of conservation of baryon number, momentum and energy) and the equation of conservation of chemical species (which includes the diffusion and the reaction rates within the combustion zone) in multidimensional numerical simulations, see 19. Due to the small width of the combustion zone in comparison with the radius of the star such a simulation is clearly numerically unfeasible.

In Ref. 10 it has been argued that such complicated simulations actually are not needed. Indeed one can divide the process of conversion of a hadronic star into a quark star into two separated regimes: i) the turbulent regime which can be described by hydrodynamics under the assumption of an infinitely thin combustion zone; ii) the diffusive regime in which the two fluids, fuel and ashes are in mechanical equilibrium, but out of chemical equilibrium. This regime is described by an advection diffusion reaction equation. The separation between the two regimes can be found by imposing the so called Coll's condition [20,21 on the thermodynamical variables of the two fluids as we will explain in the following.

\subsection{Turbulent regime}

The turbulent regime can be described within a purely hydrodynamical approach in which the combustion zone is so thin to be considered as a surface of discontinuity, the so called flame front. We will follow the treatment of Refs. [20,21 where classical combustion theory has been generalized to the framework of relativistic hydrodynamics. We indicate with $p_{i}, e_{i}, n_{i}, w_{i}=e_{i}+p_{i}$ and $X_{i}=\left(e_{i}+p_{i}\right) / n_{i}^{2}$ the pressure, energy density, baryon density, enthalpy density and dynamical volume of fluid $i$. As in the case of the discontinuity associated with a shock wave, also in the case of the flame front one imposes the continuity equations for the fluxes of baryon number, momentum and energy. By indicating with $j$ the number of baryons ignited per unit time and unit area of the flame front, the thermodynamical quantities of the hadronic fluid and of the quark fluid are related to each other by the following equations:

$$
\begin{aligned}
n_{h} u_{h} & =n_{q} u_{q}=j \\
\left(p_{q}-p_{h}\right) /\left(X_{h}-X_{q}\right) & =j^{2} \\
w_{h}\left(p_{h}, X_{h}\right) X_{h}-w_{q}\left(p_{q}, X_{q}\right) X_{q} & =\left(p_{h}-p_{q}\right)\left(X_{h}+X_{q}\right)(3)
\end{aligned}
$$

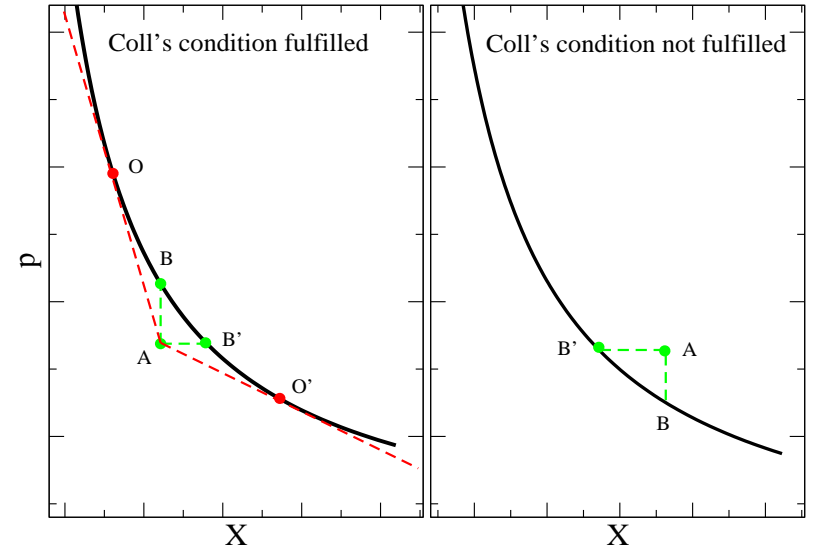

Fig. 1. Illustrative plot of the detonation adiabat in the case in which the Coll's condition is fulfilled (left panel) or not (right panel). A, B, B' indicate respectively the initial hadronic state and two possible final states for the quark phase. $\mathrm{O}$ and $\mathrm{O}^{\prime}$ are the Chapman-Jouget points. Figure taken from [10].

the last equation is the so-called relativistic detonation adiabat. $u_{h}$ and $u_{q}$ are the four-velocities of hadronic and quark matter in the flame front rest frame. If one starts from hadronic matter in a initial state $\mathrm{A}: p_{h}=p_{A}$ and $X_{h}=X_{A}$ and with a given value of $j$, Eqs. 1-3 allow to determine the final state $\mathrm{B}$ of quark matter, $p_{q}=p_{B}$ and $X_{q}=X_{B}$ which belongs to the detonation adiabat. The second equation represents a straight line in the $(\mathrm{p}, \mathrm{X})$ plane passing through A and with angular coefficient equal to $-j^{2}$. The intersections of this line with the detonation adiabat allow to find the state $\mathrm{B}$ of quark matter. The value of $j$ cannot be expressed in terms of the thermodynamical variables of the two fluids. It depends in general on the transport properties of the two fluids (the thermal conductivity and the diffusion coefficient) and the rate of chemical reactions. Therefore it must be determined within a kinetic theory approach such as the one of Ref. [13.

The so-called "condition for exothermic combustion" ("Coll's condition") for the conversion of fluid 1 into fluid 2 reads: $e_{1}(p, X)>e_{2}(p, X)$, i.e. at fixed pressure and dynamical volume, the energy density of fluid 1 , the fuel, must be larger then the one of fluid 2, the ash. As shown in Ref. 22, 10 if this condition is fulfilled the state A of the hadronic phase lies in the region of the $(\mathrm{p}, \mathrm{X})$ plane below the detonation adiabat (see left panel of Fig. 1). As a consequence, there exist two values of $j, j_{O}$ and $j_{O^{\prime}}$, for which the lines passing through A are tangent to the detonation adiabat. The two points of tangency are the Chapman-Jouget points. In particular, point $\mathrm{O}$ corresponds to the Chapman-Jouget detonation and it is the only possible realization of detonation in a physical system, such a compact star, in which no external force is producing the shock wave, see 16. If the Coll's condition is not fulfilled one cannot define the Chapman-Jouget points and the detonation combustion mode cannot take place. 
Coll's condition is important also to establish whether the deflagration combustion mode can take place. Let us consider the simplest case of a slow combustion for which the velocities $v_{h}$ and $v_{q}$ are much smaller than the sound velocities $c_{h}$ and $c_{q}$ of the two fluids. By using Eqs.(13 ) one finds in this regime that $p_{h}=p_{A}=p_{q}=p_{B^{\prime}}$ and $\left(e_{A}+p_{A}\right) / n_{A}=\left(e_{B^{\prime}}+p_{A}\right) / n_{B^{\prime}}$, i.e. the enthalpy per baryon is conserved during the combustion (see [16] for the case of non-relativistic hydrodynamics). Coll's condition implies that $X_{B}^{\prime}>X_{A}$ i.e. $\left(e_{B^{\prime}}+p_{A}\right) / n_{B^{\prime}}^{2}>\left(e_{A}+p_{A}\right) / n_{A}^{2}$ which together with the conservation of the enthalpy per baryon leads to $n_{B^{\prime}}<n_{A}$. Moreover, from $n_{A}\left(e_{B^{\prime}}+p_{A}\right)=$ $n_{B^{\prime}}\left(e_{A}+p_{A}\right)<n_{A}\left(e_{A}+p_{A}\right)$ one obtains $e_{B^{\prime}}<e_{A}$. Thus the quark phase is produced with baryon density and energy density smaller than the one of the hadronic phase: these conditions are necessary for the Rayleigh-Taylor instabilities to take place. As shown in Refs. 23, 24, the Rayleigh-Taylor instabilities do indeed occur during the conversion of a hadronic star and they substantially increase the efficiency of burning leading to time scales of the order of $\mathrm{ms}$ for the conversion of a big portion of the star 1. In Fig 2, we display one example of the dynamics of the combustion of a hadronic star during the turbulent regime. The simulation consists in solving the Euler equations in $3+1 \mathrm{D}$ by using a well-tested grid code that employs a finite volume discretization, the so-called piecewise parabolic method, see 24] and references therein. Moreover a level-set method has been used to follow the evolution of the flame front. The Rayleigh-Taylor instabilities are clearly visible (the typical mushroom structures) and render the conversion turbulent. After about $4 \mathrm{~ms}$, almost the whole star is converted and, at the same time, the turbulent eddies stop. The star has reached a configuration of mechanical equilibrium. In particular, the pressure, the energy density and the baryon density of the two phases are continuous at the interface. This is equivalent to the Coll's equality: $e_{h}(p, X)=e_{q}(p, X)$. The turbulent regime thus stops at the critical density of the hadronic phase, $\overline{n_{h}}$, for which the Coll's equality is satisfied. For $n_{h}<\overline{n_{h}}$, the Coll's condition is violated. This implies that the new phase is produced with $e_{B^{\prime}}>e_{A}$ and thus the hydrodynamical instabilities causing turbulence cannot anymore take place. Notice however that at the interface the temperature and the chemical of the two fluids are discontinuous. Therefore the burning can proceed but with velocities which are dominated by the diffusion and the rate of the

\footnotetext{
${ }^{1}$ Notice that our framework is similar to what in the literature is known as pre-mixed combustion. The distinction between a premixed and non-premixed scheme is related to the value of the diffusive burning velocity. If that velocity is very large, as suggested in Ref. 14 then the increase of the velocity due to turbulence is marginal (if any at all). This is close to the result of Ref.24 (see Fig.7) because in that paper the laminar velocity estimated in 14 has been adopted for the numerical simulations. In our scheme, we are instead using the velocities estimated in 13.15 which are significantly smaller and the increase of the velocity due to turbulence is much larger. This is due to the dependence of the Gibson scale on the laminar velocity.
}

chemical reactions and which are much smaller than the velocities obtained during the turbulent regime. A natural question arises: is the process still exothermic during the diffusive regime? As discussed before (see also Ref.[16]), a slow combustion is characterized by the continuity of the pressure and the enthalpy per baryon across the combustion front. Those two continuity conditions allow to compute the state of the newly produced quark matter. We have numerically solved these equations in [10] and we have verified that the new phase is produced at a temperature higher than the temperature of the fuel. This is actually the condition of exothermicity because it implies that some heat will be released from the star because of the conversion. It is interesting to notice that an analytic argument can be provided to show that the conversion remains exothermic till the surface of the star, see [10].

\subsection{Diffusive regime}

Let us now discuss how do we model the subsequent evolution of the conversion during the diffusive regime. First, we need an initial density profile of the star after the turbulent regime: this configuration is composed by hot quark matter for densities larger than $\bar{n}_{h}$ and by cold hadronic matter for densities smaller than $\bar{n}_{h}$ (we are discussing here the case of the conversion of cold hadronic stars). The EoS of hot quark matter is computed by requiring that at fixed pressure, the enthalpy per baryon of the quark phase is equal to the one of the hadronic phase as in the case of a slow combustion. The underlying hypothesis here is that the kinetic energy of the turbulent eddies taking place during the first stage of conversion dissipates into heat. Notice that since the turbulent regime lasts few ms, neutrino cooling (occurring on time scales of seconds) is not active during the first stage of the conversion. In Fig. 3. we show one example for the configuration of a $1.5 M_{\odot}$ hadronic star which contains hyperons (black lines) which has undergone the turbulent conversion into a star almost entirely composed by quark matter (red lines). The upper panel displays the mass enclosed and the lower panel the radius as functions of the baryon density. Notice that after the turbulent regime (the density at which this regime stops is indicated by the red dashed line) a mass of about $0.5 M_{\odot}$ remains unburnt within a layer with a thickness of about $3 \mathrm{~km}$.

The dynamics of the diffusive regime is regulated by two differential equations, one describing the propagation of the flame front and the other describing the thermal evolution of the star in presence of the neutrino cooling process and taking into account the heat gradually released by the conversion of the layers left unburnt during the turbulent regime. Concerning the position of the flame front, by labeling with $r_{f}(t)$ its radial coordinate, one can write:

$$
\frac{\mathrm{d} r_{f}}{\mathrm{~d} t}=v_{l f}\left(\mu_{q}, T\right)
$$

where $v_{l f}$ is the laminar velocity of the front with respect to the quark matter fluid (see [10]). The initial condition 


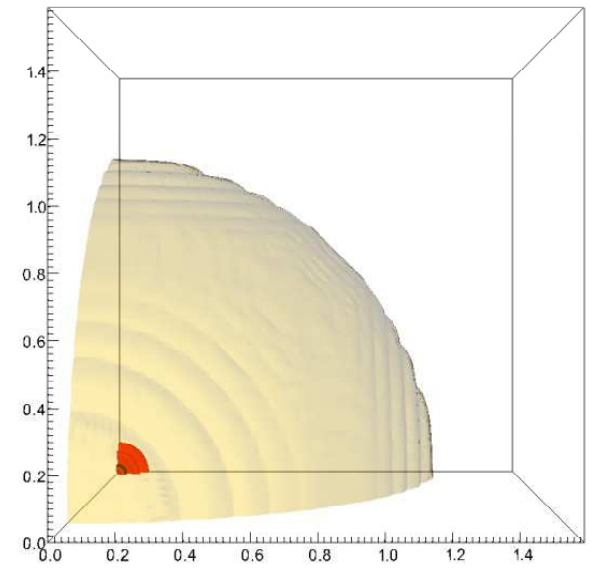

(a) $t=0$

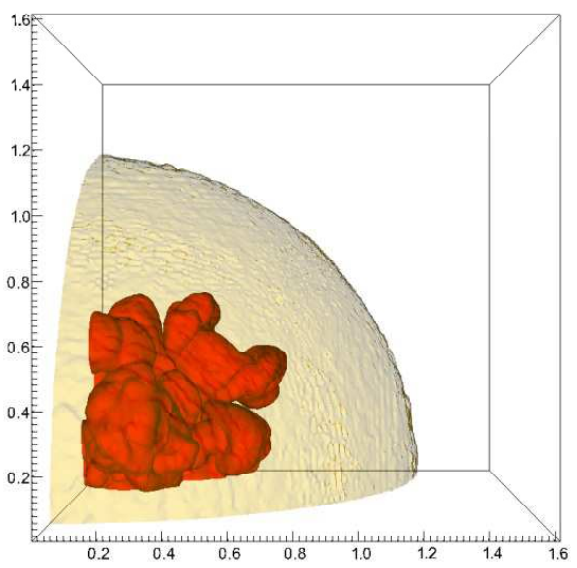

(c) $t=1.2 \mathrm{~ms}$

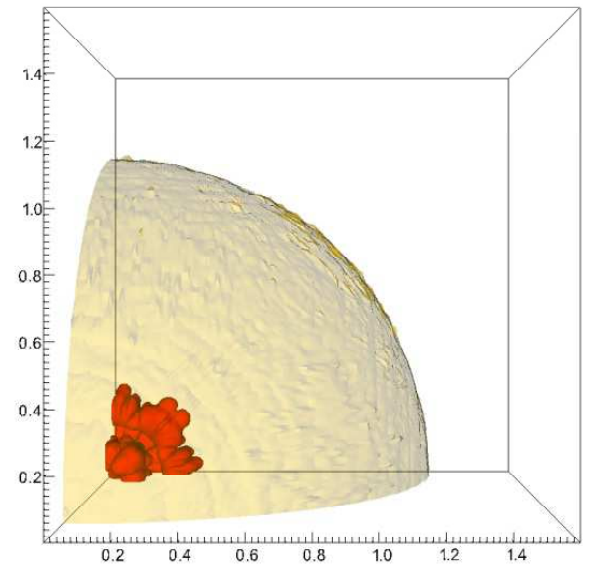

(b) $t=0.7 \mathrm{~ms}$

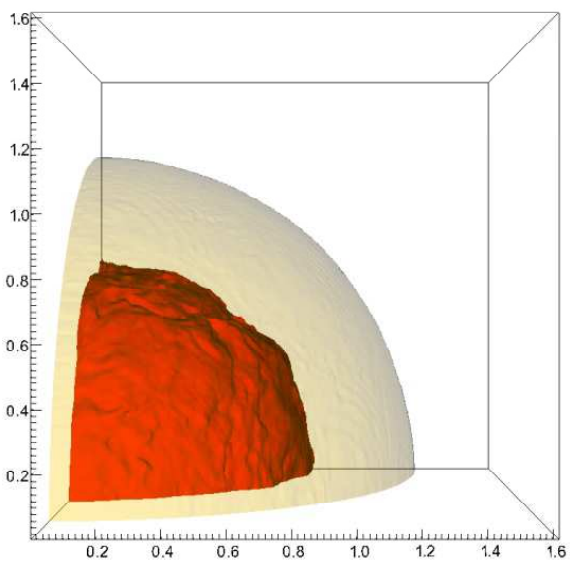

(d) $t=4.0 \mathrm{~ms}$

Fig. 2. (color online) Conversion front (red) and surface of the neutron star (yellow) at different times $t$. Spatial units $10^{6} \mathrm{~cm}$ taken from [25].

reads $r_{f}(0)=\bar{r}$ where $\bar{r}$ is obtained from the baryon density profile by using the equation $n_{h}(\bar{r})=\overline{n_{h}}$. The thermal evolution is in principle very complex since one should consider how the heat progressively generated in the conversion of the external layers is distributed within the star and one should implement a diffusion transport code for handling the propagation of neutrinos. This last task has been treated in Ref. 25 for the configuration obtained just after the turbulent regime but without considering the further conversion of the star in the diffusive regime. A sensible approximation is to consider the thermal evolution of the star as being dominated, during the first few seconds, by the diffusion of the heat deposited during the rapid burning of its central region (the burning and the cooling of the external layer is sub-leading). After this period of time the external layers of the star are almost isothermal 25] therefore we can make the simplifying assumption that in the subsequent evolution within the diffusive regime the star is basically isothermal. The simple picture is then the following: the flame propagates towards the surface and releases the heat of the conversion; the neutrino cooling operates via a black body surface emission with a corresponding luminosity $L=21 / 8 \sigma(T / K)^{4} 4 \pi r_{s}^{2}$ $\mathrm{erg} / \mathrm{s}$ [26] with $r_{s}$ the radius of the neutrinosphere (we will assume that it is located at the interface between the inner crust and the outer crust) The thermal evolution equation then reads:

$$
C(T) \frac{\mathrm{d} T}{\mathrm{~d} t}=-L(T)+4 \pi r_{f}^{2} j\left(r_{f}, T\right) q\left(r_{f}, T\right)
$$

where $C$ is the heat capacity of the star, $L$ the neutrino luminosity, $j$ is the number of baryons ignited per unit time and unit area and $q$ is the heat per baryon released by the conversion. Concerning the heat capacity, we use $C=2 \times 10^{39} M / M_{\odot}\left(T / 10^{9}\right) \mathrm{erg} / \mathrm{K}$ obtained in Ref. [26] for a uniform density quark star or a hadronic star.

By solving simultaneously Eqs. 4 and 5 with initial conditions: $r(0)=\bar{r}, T(0)=T_{0} \mathrm{MeV}$ (which is the temperature of the star for $r>\bar{r}$ after the turbulent regime 
and it is of the order of $5 \mathrm{MeV}$ as found in 25]) we can calculate the time needed to complete the conversion of the star and the neutrino luminosity due to the conversion of the material left unburnt after the turbulent stage. In Fig 4, we show three cases corresponding to different values of the parameter $a_{Q *}^{\max }$ which is related to the minimum amount of strangeness needed to render the conversion process exothermic and enters in the expression of $v_{l f}$, see [10. We also show a curve of luminosity corresponding to a simple exponential parametrization of the neutrinos released from the heat generated during the turbulent regime: $L(t)=Q / \tau e^{-t / \tau}$ with $\tau \sim 3$ s and $Q \sim 8.5 \times 10^{52}$ erg (see Fig 5 for the luminosities computed in [25].)

A remarkable feature is that during the diffusive regime the neutrino luminosity displays a quasi-plateau (particularly evident for the smallest value of $\left.a_{Q *}^{\max }\right)$. This feature is related to the scaling of the burning velocity with the temperature: $v_{l f} \propto T^{-5 / 6}$. As the conversion proceeds, the temperature increases due to the release of energy and therefore the velocity decreases. It is a self-regulating mechanism which rapidly leads to an almost constant velocity of burning and an almost constant luminosity of neutrinos. The process goes on until the whole star is converted. The kink appearing in the luminosity curves signals the end of the conversion: the following evolution is governed only by the cooling and the standard power law luminosity is obtained. Typical time scales to complete the conversion, in this specific case, are of the order of few tens of seconds. Actually these times scales can be reduced by considering that, due to gravity, the external layer will tend to fall onto the conversion front as the flame propagates. This would lead to an acceleration of the front which reduces the time of the conversion by roughly a factor of three/four 2 .

\section{Long GRBs}

GRBs are divided into two subclasses, long GRBs, having a duration of more than $2 \mathrm{~s}$, and short GRBs, lasting less than $2 \mathrm{~s}$ 27,28. This division is clearly schematic and one cannot rule out the possibility that elements of one class intrude the other. The characteristics of the GRBs in the two classes should derive from the different astrophysical scenario at their origin. Short GRBs are generally assumed to be generated by the merging of two compact stars in a binary system. We will discuss them in the next Section. The origin of long GRBs instead is typically associated with the collapse of one massive star, either forming a black-hole (collapsar model [29,30]) or forming a millisecond proto-magnetar, a model also known as evolutionary wind model 31. At the moment it is not obvious if all long GRBs should be produced by only one of the two proposed models, or if both possibilities are realized in Nature, under different initial conditions of the collapsing star. In particular, the proto-magnetar model requires very strong magnetic fields, of the order of $10^{15} \mathrm{G}$ and a rotation period of the newly formed magnetar of the order

${ }^{2}$ Drago and Pagliara, work in progress.

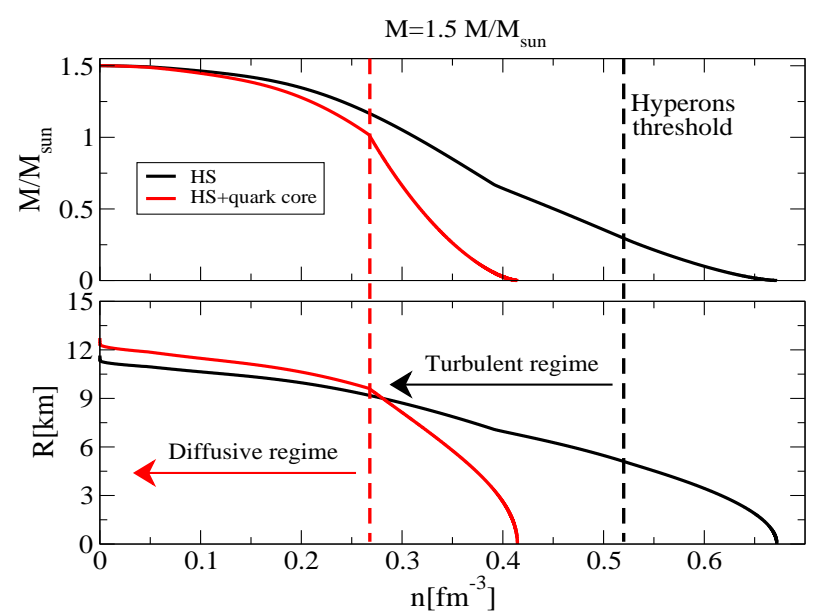

Fig. 3. Enclosed gravitational mass and radius as a function of the baryon density for a $1.5 M_{\odot}$ hadronic star before the turbulent conversion (black lines) and after the turbulent conversion (red lines). The black dashed line marks the appearance of hyperons: the seed of strange quark matter is formed at densities larger than this threshold. The red dashed line marks the density below which Coll's condition is no more fulfilled and the turbulent combustion does not occur anymore. Below this density, the combustion proceeds via the slow diffusive regime. Figure taken from 10.

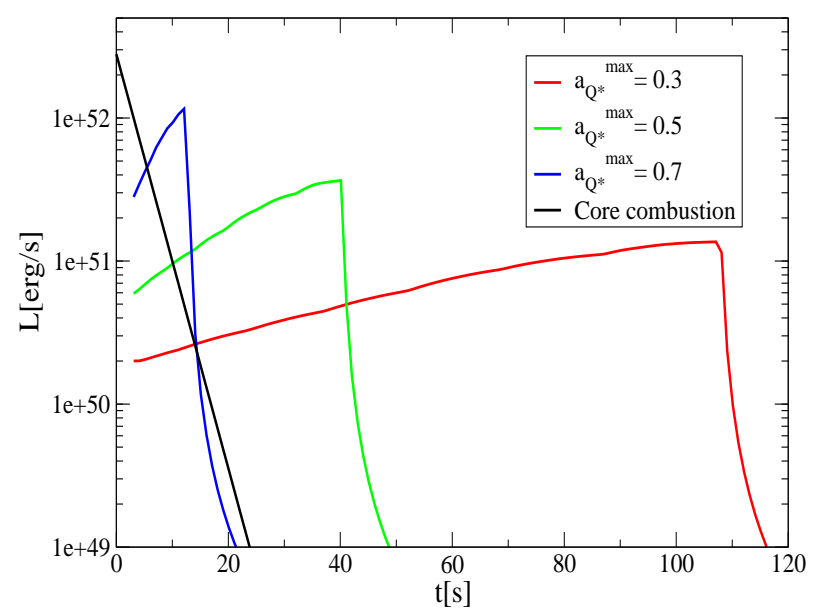

Fig. 4. Neutrino luminosity associated with the burning during the diffusive regime of the combustion for three choices of the parameter $a_{Q *}^{\max }$. The black line represents the luminosity obtained from the rapid combustion of the core. Figure taken from [10.

of a millisecond. It is not obvious how easy these two conditions can be reached and the possibility that, at least in a few cases, some GRB is generated by the collapsar model is still open, even though long GRBs associated with a SN are probably compatible with the proto-magnetar model and not with the collapsar's one [32].

In this review we will shortly discuss both possibilities and we will see under which conditions quark deconfine- 


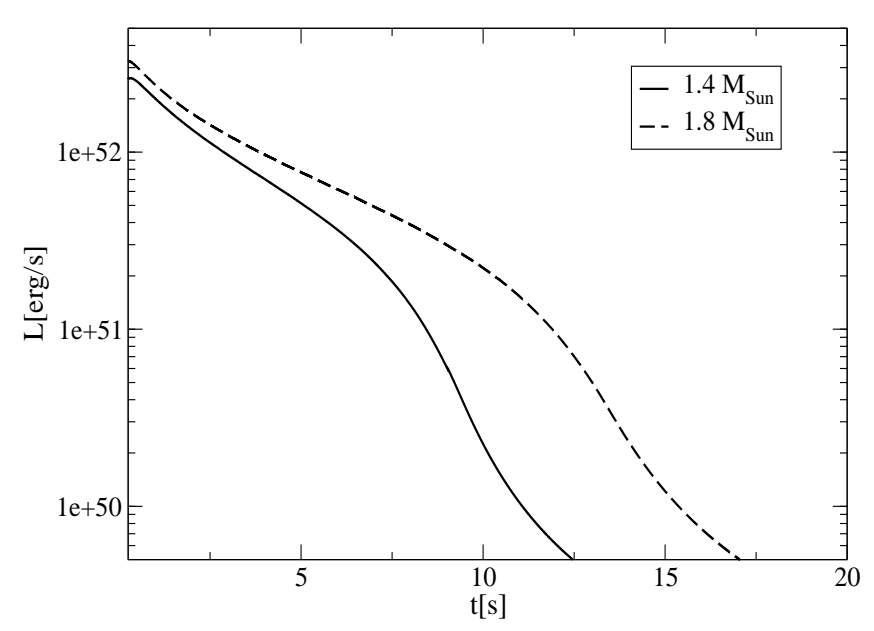

Fig. 5. Total neutrino luminosity as a function of time. The two curves refer to the turbulent (and thus not complete) conversion of a $1.4 M_{\odot}$ and a $1.8 M_{\odot}$ stars. Figure taken from 25 .

ment can play a role and produce some observable signature.

\subsection{Models for long GRBs}

Any realistic model for the inner engine of long GRBs should take into account a few basic findings:

- the total energy emitted in x-rays and in $\gamma$-rays is large, of the order of $10^{50}-10^{51} \mathrm{erg}$;

- the typical duration of the initial very luminous phase, called prompt emission, is of the order of a few tens of seconds, although much longer durations have been observed in a few cases:

- in a significant fraction of long GRBs a prolongated emission has been observed, lasting up to $10^{3}-10^{4} \mathrm{~s}$. While its luminosity is much lower than that of the prompt emission the total energy emitted during this "quasi-plateau" phase is not much smaller than the energy emitted during the prompt phase;

- the photons observed during the prompt phase can be well described if one assumes that they are produced by internal shocks of a ultrarelativistic plasma, expanding with a Lorentz factor $\Gamma$ of the order of $10^{2}-10^{3}$

- the rapid variations in the luminosity of the prompt phase, taking place on a submillisecond scale, imply that the source has to be compact;

- the position of the source has been located in a few cases and it corresponds to a star formation area of the host-galaxy 33].

All these data suggest that the inner engine of long GRBs is a collapsing massive star and that in many cases some activity still exists $10^{3}-10^{4} \mathrm{~s}$ after the collapse.

The main difference between the two models lies in the ultimate source of the energy used to produce the burst: in the collapsar model one uses the energy in the accretion disk around the black-hole (in principle one can also use the energy of the rotating black-hole), extracted by the neutrinos. Instead, in the evolutionary wind model the source of the energy is the rotational energy of the protomagnetar. As we will see, in both cases one can imagine that quark deconfinement can be used to modify the model and the energy associated with the phase transition can be used to power a burst.

\subsubsection{The collapsar model}

The central idea behind the collapsar model is rooted into the ultimate fate of very massive stars 34, in particular stars having a mass larger than about $25-30 M_{\odot}$ and whose external hydrogen and helium layers have been lost due to strong winds (Wolf-Rayet stars), see Fig. 6. The main points of the model are the following:

- the progenitor starts collapsing. A failed supernova follows and a black-hole forms either directly or due to the large fallback ;

- an accretion disk forms. If the angular momentum in the disk is appropriate most of the energy in the disk can be extracted by neutrino-antineutrino emission [29];

- due to the toroidal geometry neutrino-antineutrino annihilation is a rather efficient mechanism and a plasma of electrons and positrons forms in the area around the black-hole;

- the rotation of the progenitor allows the formation of a empty channel along the rotation axis (funnel) on a time scale of the order of ten seconds;

- the electron-positron plasma can escape the cocoon of the progenitor along the funnel. In that way a collimated jet can also form;

- a fraction of the energy of the jet can also be used to repower the supernova producing a successful explosion of the Ic type.

The model is very predictive and this lead initially to spectacular confirmations and more recently, with more precise data, to some possible problems. In particular the association between GRBs and Ic SNae has been confirmed in a few cases $35,36,37$. On the other hand one problem appeared: the energy of the associated SN has an energy of about $10^{53} \mathrm{erg}$, much larger than the energy of the jet, what makes the idea of a SN revitalized by the GRB difficult to justify. The energy of the SN on the other hand is similar to the rotational energy of a millisecond pulsar, what can be a strong argument in favor of the proto-magnetar model [36. There is also another possible problem: at least in one case no associated SN has been observed [38. Finally, in a significant number of cases the prompt emission is made of two well separated active periods (3 active periods have been observed only in one case), with a long quiescent time in between. It is still not clear if there is a statistical evidence of an excess of bursts having long quiescent times respect to the distribution of all intervals (long or short) separating the active phases. In a few papers in the past, that evidence was apparently found 39,40, but a recent re-analysis 41 


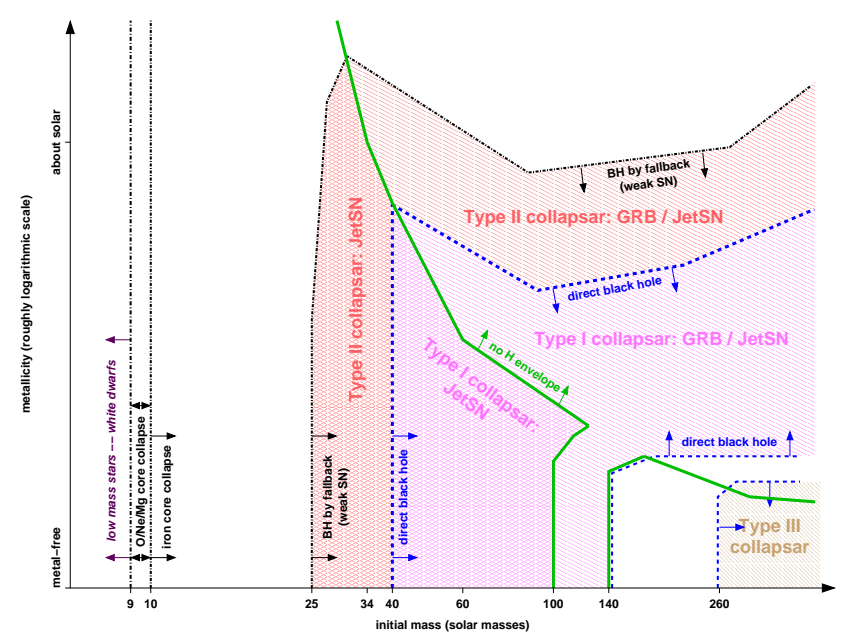

Fig. 6. Collapsar types resulting from single massive stars as a function of initial metallicity and initial mass. The main distinction is between collapsars that form from fallback (Type II; red) and directly (Type I; pink). One can subdivide these into those that have a hydrogen envelope (cross hatching), only able to form jet-powered supernovae (JetSNe) and hydrogenfree collapsars (diagonal cross hatching), possibly making either JetSNe or GRBs. The first subclass is located below the thick green line of loss of the hydrogen envelope and the second is above it. The light brown diagonal hatching at high mass and low metallicity indicates the regime of very massive black holes formed directly (Type III collapsars) that collapse on the pair-instability and photo-disintegration. Since the collapsars scenario require the formation of a $\mathrm{BH}$, at low mass (left in the figure) or high metallicity (top of the figure) and in the strip of pair-instability supernovae (lower right) no collapsars occur (white). Figure taken from [34].

indicates that maybe all inter-peaks durations can be described by using a same statistical distribution. On the other hand it is not trivial to explain quiescent times of the order of minutes just assuming that they are due to some statistical fluctuation: a more detailed description of how they do take place seems mandatory.

The collapsar model has also some difficulties in explaining the long emissions taking place, in many cases, after the prompt emission. While the idea of debris still collapsing onto the black-hole on that long time-scale cannot be completely ruled-out it seems difficult to justify considering the regularity of the emission.

\subsubsection{The proto-magnetar model}

The central idea behind the evolutionary wind model is that at the origin of a long GRB there is a successful SN producing a rapidly rotating magnetar [31. The sequence of the events in the model is the following, see Fig 7
- after the SN explosion a magnetar forms, with a magnetic field of the order of $10^{15} \mathrm{G}$ and a period of about one millisecond;

- the magnetar starts cooling down by emitting neutrinos and antineutrinos;

- whatever charged material is ejected from the star it is strongly accelerated by the large Poynting flux $\dot{E}$ due to the enormous magnetic field and the very rapid rotation;

- the strong neutrino emission ablates material from the surface of the star. For some ten seconds the baryon flux is so large that the forming jet has a low Lorentz factor due to the baryonic contamination;

- the neutrino luminosity reduces and similarly the baryonic flux. The Poynting flux remains almost constant and a jet with a $\Gamma \sim 10^{2}-10^{3}$ can form. These are the right conditions to generate strong internal shocks in the expanding plasma: they are at the origin of the observed emission;

- the baryonic flux further reduces and the jet becomes almost baryon free. Under these conditions the motion of the particles in the plasma is almost collinear and internal shocks are suppressed. The prompt emission terminates;

- the magnetar is still rapidly rotating (although less rapidly) and a pulsar-type emission can take place, explaining the long quasi-plateau phases [42,43].

This model has many interesting features: the most important one is that it allows to explain the order of magnitude of the energy of the associated SN explosion: it is the rotational energy of the magnetar. Another extremely nice feature is that it explains in a very natural way the quasi-plateau emissions, as due to a pulsar-like activity. It is indeed possible to model in a very precise way all the quasi-plateau just by fitting two numbers: the magnetic field and the rotation's period [42,43. It has one possible weak point: the maximum Lorentz factor $\sigma_{0}$ first increases due to the reduction of the baryonic flux and then slowly decreases, due to the gradual slow-down of the rotation of the star. It is therefore impossible to explain within the model the temporal structure of GRBs having two active periods separated by minutes of quiescence. We will see how quark deconfinement can solve that problem. Also, the model predicts that all GRBs are associated with a successful SN, while at least in one case no SN has been observed. Again quark deconfinement can provide a possible explanation.

\subsection{The role of quark deconfinement}

As discussed in the previous subsections, there are two problems which are quite difficult to solve either in the collapsar or in the proto-magnetar model. The first is associated with GRBs displaying a second peak in the prompt emission, separated from the first peak by a long quiescent time. The second problem is the possible existence of GRBs not associated with a SN explosion. In the following we discuss these two problems and we show how they can be solved in the two-families scenario. 


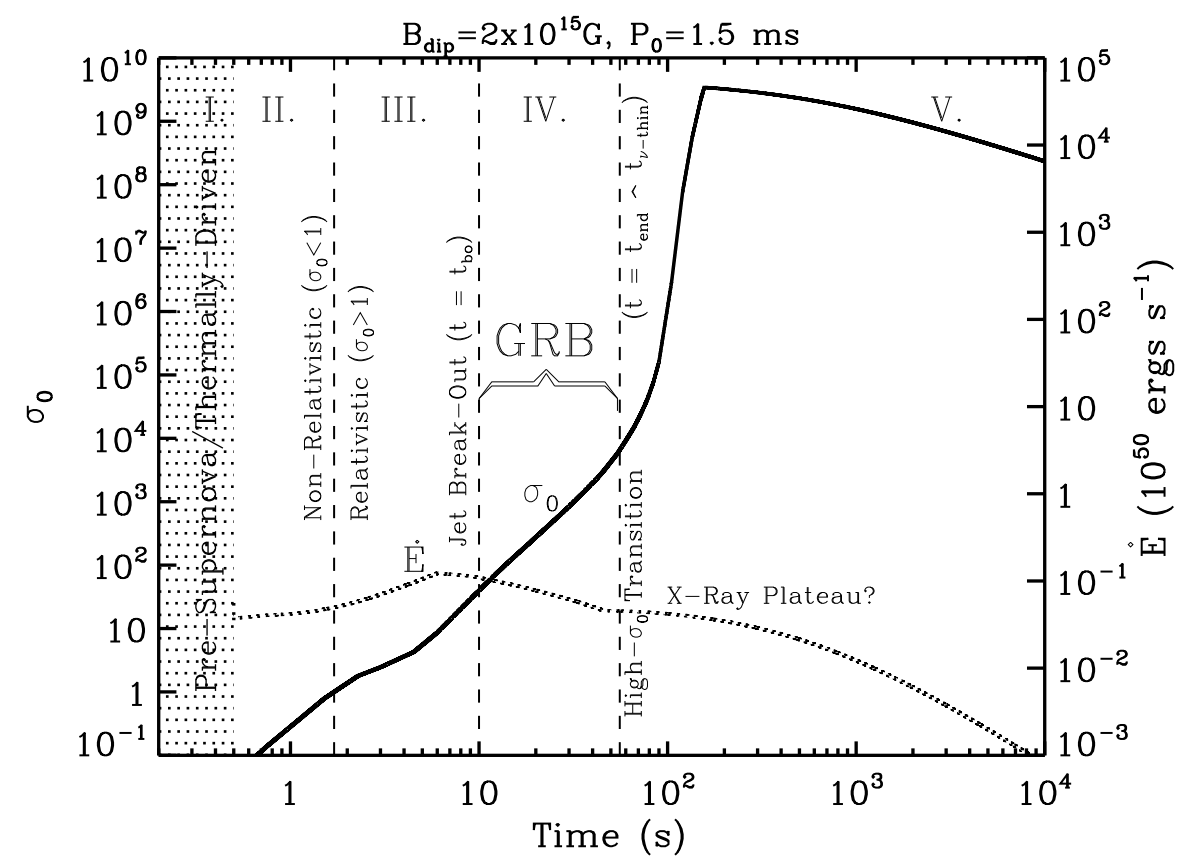

Fig. 7. Wind power $\dot{E}$ (right axis) and magnetization $\sigma_{0}$ (left axis) of the proto-magnetar wind as a function of time since core bounce, calculated for a neutron star with mass $M=1.4 M_{\odot}$, initial spin period $P_{0}=1.5$ ms, surface dipole field strength $B_{\text {dip }}=2 \times 10^{15} \mathrm{G}$, and magnetic obliquity $\chi=\pi / 2$. Figure taken from [31].

\subsubsection{Long quiescence times}

In a few cases GRBs display a prompt emission composed of two events separated by a period of quiescence which can be very long. The most spectacular case is the one of GRB 110709B 44] (see Fig 8) in which the two events are separated by more than ten minutes. The two emissions present similar luminosities and light curve characteristics (although with a different time evolution of the spectral properties). Interestingly, GRBs presenting more than two well separated events are very rare and probably the only relevant example is that of GRB 091024 [45] presenting three episodes of comparable emission. The question is therefore how to justify what seems a case of reactivation of the inner engine.

The statistical analysis of Refs. 39, 40, indicated an excess of long quiescence times respect to a log-normal distribution fitted to reproduce inter-pulse durations of whatever length. Those analysis were therefore suggesting a possible different physical origin for the long quiescence times. A very recent reanalysis on the other hand seems to indicate that when the peak detection efficiency is taken into account the log-normal distribution has to be substituted with a power-law which is able to describe the waiting-time distribution of all the pulses. The authors of [41] are therefore suggesting that the pulses are due to the fragmentation of the accretion disk, within the collapsar model. While that model seems good at interpreting the distribution of the waiting-times, at least two possibly connected questions remain open:

- the analysis performed in 39] indicates that on the average the second episode lasts twice as long as the first one: GRB 110709B is just one representative example of that situation;

- explicit simulations are not suggesting that, if the disk fragments, the inner part (the one powering the first episode) is smaller than the second one.

Within the two-families scenario it is rather natural to interpret the second episode as due to quark deconfinement within the proto-magnetar model. The possible scheme is the following:

- the first episode of the long GRB is generated exactly as described with the proto-magnetar model: the baryons are ablated from the surface of the compact star by the neutrinos associated with the cooling of the newly formed compact star whose temperature was of about $20-30 \mathrm{MeV}$ immediately after the collapse;

- the star starts slowing down (the initial rotation period is of the order of the millisecond) and therefore its central density increases;

- if the central density of the star during the first episode was slightly below the critical density needed to deconfine the quarks than during the process of slow-down the critical density can be reached;

- the process of quark deconfinement is strongly exothermic and the inner temperature of the star increases again up to a temperature comparable to the one reached before;

- baryons are again ablated from the surface of the forming quark star, as long as the surface is not completely converted into quark matter: a new episode of the GRB can therefore take place. 
This scheme presents at least a couple of delicate points that will need to be examined in details in the future. The first point is the neutrino emission during deconfinement. As we have seen in Section II the neutrino luminosity due to deconfinement has two components: one is associated with the cooling of the central area of the star which has deconfined very rapidly, the other is associated with the heat deposited in the outer part of the star while the process of deconfinement keeps going till it reaches the surface. The first component can be approximated as $L_{\nu}^{c} \sim Q / \tau_{\text {diff }} \exp \left(-t / \tau_{\text {diff }}\right)$. In the case of long GRBs the heat $Q$ deposited during the rapid deconfinement corresponds roughly to half of the total deconfinement energy $\Delta E$ of a compact star having a initial mass of about (1.4$\left.1.5 M_{\odot}\right) . \Delta E \sim 0.15 M_{\odot} \sim 4.5 \times 10^{53} \mathrm{erg}$ (see paper 1 ) and therefore $Q \sim 2 \times 10^{53} \mathrm{erg}$, while $\tau_{\text {diff }} \sim(2-3) \mathrm{s}$. The typical neutrino energy is about $10 \mathrm{MeV}$. After some ten seconds, the luminosity of the neutrinos associated with the cooling of the central area becomes comparable with that associated with the deconfinement of the outer region and, more importantly, it becomes sufficiently low to allow the possibility of having $\Gamma \sim 10^{2}-10^{3}$ if the Poynting flux $\dot{E}$ remains similar to the one of the first event.

The second delicate point concerns the evolution of the Poynting flux. One peculiarity of the two-families model is that the quark star formed after the transition has a larger radius and therefore a larger moment of inertia than the hadronic star before the transition. Therefore there is a rather strong reduction of the angular velocity during the transition and this implies a strong reduction of the Poynting vector unless the magnetic field increases at the same time. The behavior of the magnetic field during a quark deconfinement phase transition can be quite complicated. Buoyancy forces can move an internal toroidal magnetic field towards the surface and quark deconfinement can help by reducing the anti-buoyancy forces 46 47.48. Since the internal magnetic field is typically larger than the external one it is possible that during the process of quark deconfinement the external magnetic field increases. In that way the Poynting flux could remain more or less constant. Clearly at the moment these are little more than speculations and will need to be addressed in future calculations.

\subsubsection{GRBs not associated with a SN}

Both the collapsar's and the proto-magnetar model are based on a strict association between a long GRB and a $\mathrm{SN}$ explosion. Indeed in a few cases a type Ic SN has been found associated with the GRB. One can also imagine that the more the GRB is far away the more it is difficult to detect the associated SN. On the other hand at least one case exists, GRB 060614 [38,49,50, of a close-by GRB for which no associated SN has been observed. This suggests the possibility of GRBs generated through a mechanism not involving a SN explosion 3 . The merger of two NSs is

\footnotetext{
${ }^{3}$ It is also possible that the associated $\mathrm{SN}$ is sub-luminous because of the fallback of ${ }^{56} \mathrm{Ni}$ onto the $\mathrm{BH}$ see Ref. [51].
}

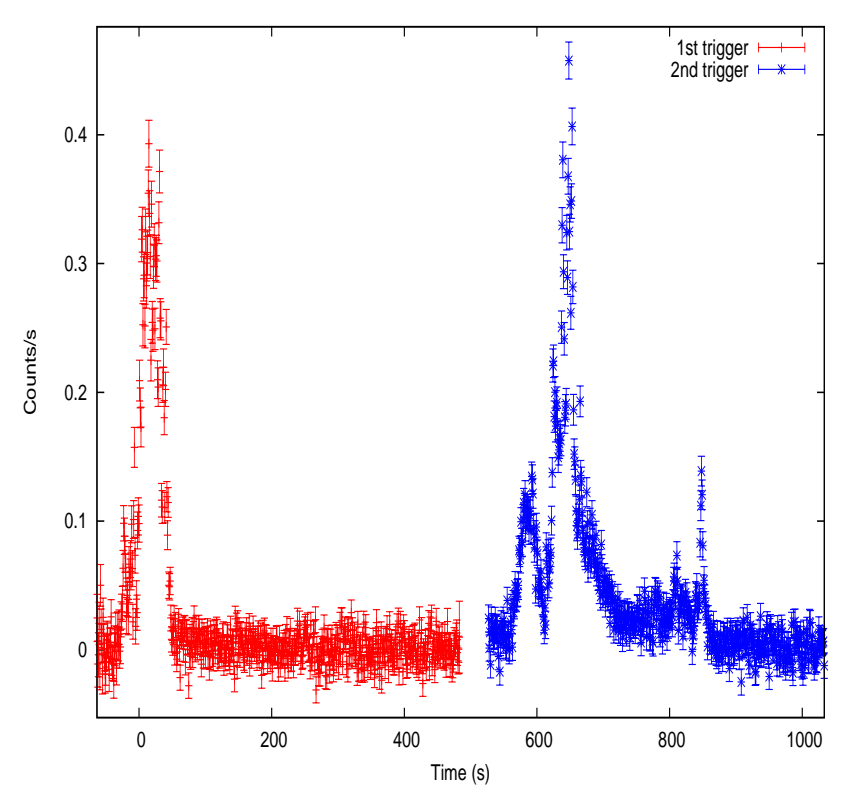

Fig. 8. BAT count rates of GRB 110709B.

such a possibility, but it is associated with short GRBs, as we will discuss in the next session. Here we instead consider the possibility of a phase transition triggered by mass accretion onto the neutron star in a LMXB system. In the two families scenario it is rather easy to have quark deconfinement at the end of mass accretion 2 and that transition deposits about $4.5 \times 10^{53} \mathrm{erg}$ of heat in the compact star. Still, to generate a strong GRB one needs to transform that heat into a plasma made mainly of electronpositron pairs and of photons and to collimate the jet. One possibility is to consider neutrino-antineutrino annihilation, a not very efficient process in the case of spherical symmetry (it is difficult to have head-on collisions between the neutrinos) so to produce a plasma of about $10^{50}-10^{51}$ erg. The magnetic field (which in accreting LMXBs cannot be larger than about $10^{10} \mathrm{G}$ ) and the rapid rotation would then beam the plasma, generating a burst that is presumably less energetic and less collimated than a typical GRB. The ultimate source of the energy of this burst is the one deposited in the plasma by neutrino-antineutrino annihilation.

Another possibility is to assume that the neutron star merges with the white dwarf. It has been shown that this process produces a spinning Thorne-Zytkow-like object with a low temperature, $T \sim 10^{9} \mathrm{~K}[52$. If large magnetic fields are generated, for instance via magneto-rotational instabilities, the conditions for producing a powerful GRB are fulfilled. Such a burst would be similar to a short GRB because it is associated with the merger of two compact stars, but its duration would be comparable with the one of long GRBs. These features are in agreement with the properties of GRB 060614. 


\section{Short GRBs}

Short Gamma-Ray Bursts are characterized by durations typically not longer than about $2 \mathrm{~s}$, and they are assumed to be associated with the merger of compact stars (NS-NS or NS-BH) in binary systems [28].

While short GRBs were discovered through their luminous prompt emission (similarly to what happened in the case of long GRBs) an extended emission was later found to exist in a significant fraction of short GRBs 53 . It was generally assumed that the prompt emission of short GRBs is spectrally harder than the one of long GRBs, but the differences are less evident when the sample is restricted to short GRBs with the highest peak fluxes 54 or when considering only the first $\sim 2 \mathrm{~s}$ of long GRBs light curves. This was summarized in Ref. 55 by saying that when comparing the prompt emission of short GRBs and the first seconds of long's, one finds: (i) the same variability, (ii) the same spectrum, (iii) the same luminosity and (iv) the same $E_{\text {peak }}-L_{\text {iso }}$ correlation. In other words, if the central engine of a long GRB would stop after $\sim 0.3 \times(1+z)$ seconds the resulting event would be indistinguishable from a short GRB 56 .

The similarities between long and short GRBs are not limited to the prompt emission: actually by comparing the quasi-plateau of long GRBs and the extended emission of short GRBs one discovers that they can both be described by assuming that a proto-magnetar formed, rotating with a period of the order of a few milliseconds and by associating the prolongated emissions to the pulsar-like emission of that object [57. The rotation period requested is in both cases of a few milliseconds, the magnetic field is of the order of a few $10^{15} \mathrm{G}$ for the long and roughly one order of magnitude larger for the shorts, see Fig.9.

A recent analysis 58 suggests that short GRBs can be classified in three categories: a) those without any extended emission; b) those with an extended emission followed by a rapid decay of the luminosity; c) those with an extended emission slowly decaying. They propose to associate the three cases to: a) formation of a $\mathrm{BH}$ soon after the merging; b) formation of a supramassive star collapsing into a $\mathrm{BH}$ after having lost part of its angular momentum; c) formation of a very massive and stable compact star after the merging. In this way they also estimate the mass distribution of the post-merger remnant as $2.46_{-0.15}^{0.13} M_{\odot}$. Although this distribution includes also supramassive stars, it indicates that very massive compact stars do exist.

A question naturally arises: if both the long and the short GRBs can be explained, at least in a fraction of cases, by assuming that a proto-magnetar forms, with similar values for the rotation period and for the magnetic field, why then the prompt emission of long GRBs lasts tens of seconds and those of shorts tenths of seconds? In both cases the ablation of material from the surface of the proto-magnetar, due to neutrino cooling, will provide the crucial ingredient to generate a jet with the proper Lorentz factor. The cleaner environment and the higher temperatures [3] reached after the merger respect to the post-supernova case would suggest that the duration of

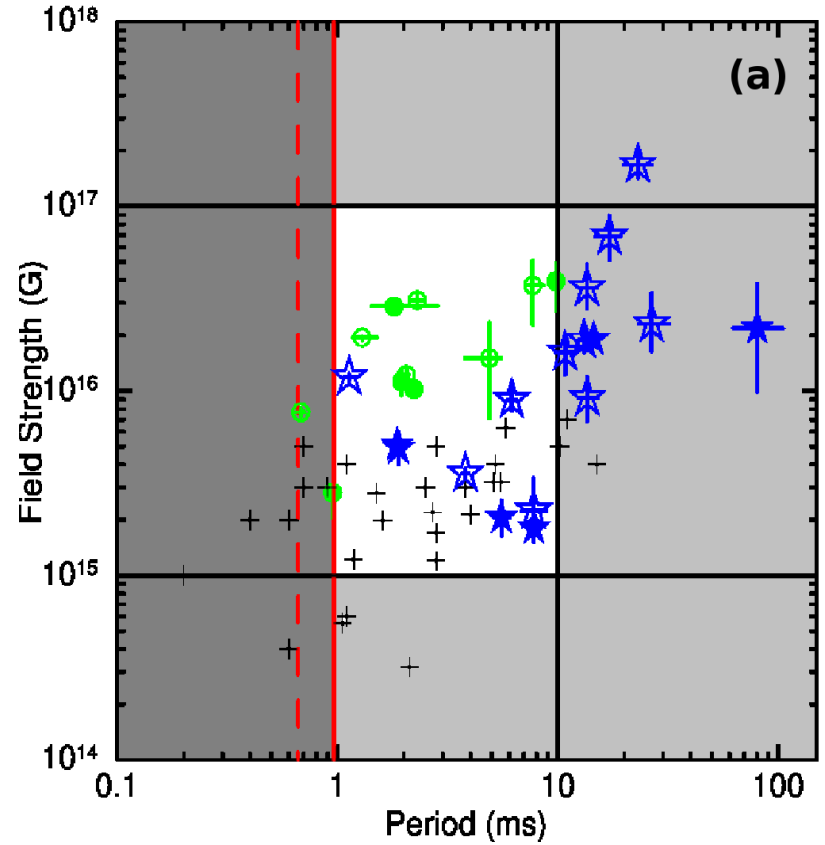

Fig. 9. Magnetic field and spin period of the magnetar fits to the extended emission of both long (black "+") and short GRBs. The latter are further separated in stable magnetars (blue stars) and unstable magnetars collapsing to form a BH (green circles). Figure taken from [57].

the short should be at least comparable to the one of the long. Which is then the mechanism stopping the prompt emission in the case of short GRBs? In the next subsection we will show how quark deconfinement can play the crucial role in this situation.

\subsection{Duration of short GRBs and quark deconfinement}

One of the best known properties of quark stars is that once formed it is impossible to ablate hadrons from its surface (unless by neutrinos having energies exceeding about $1 \mathrm{GeV})$. This is due to the confinement of quarks which does not let them to be ejected if not inside a colorless object as a hadron. A cumulative transfer of energy and momentum to a single quark by multiple neutrino scattering would also not allow to produce a hadron, because that four-momentum is rapidly shared with the other quarks by strong interactions (a similar idea has been discussed in Ref. [59]). This property of quark stars opens the possibility of explaining the rapid truncation of the prompt emission of short GRBs. Notice that in the two-families scenario, if a compact star (and not a $\mathrm{BH}$ ) forms after the merging, it is unavoidably a quark star.

In Ref. 60] the following scheme has been developed:

- a few milliseconds after the merging, the conditions are favourable for the formation of deconfined quark matter in the hot and rapidly rotating compact object;

- following the scheme described in Sec2 in a few milliseconds the central region of the star converts into 
quark matter. The new object, made of an inner part of stiff quark matter and of an external part made of hadrons is mechanically stable (although not chemically stable, since it keeps converting into quarks);

- in some ten seconds the star entirely converts into a quark star: until that moment it still has a surface made of nucleons which can be ablated. Since the star is still very hot the baryon flux during that first stage is too large to allow the formation of a jet with a large enough Lorentz factor;

- once the surface of the star has completely converted into quarks, baryons can no more be ablated and the possibility of generating a GRB no more exists;

- the GRB can be produced only during the short time associated with the the switch-off of the baryon flux. The time needed to convert the entire surface of the rapidly rotating (and therefore strongly deformed star) plays a fundamental role in regulating the duration of the GRB.

\section{Supernovae}

The possible influence of quark deconfinement on SN explosions has been explored in a few papers, starting from Refs. 61, 62. In those papers it was assumed that quark deconfinement takes place before deleptonization (QDBD following the notation of Ref. 63 ), either at the moment of the collapse or a fraction of a second after, when material falls back due to the failure of the SN explosion. Since in the mixed phase the adiabatic index is very low, the collapse continues rapidly through the mixed phase till the central density reaches the second critical density separating mixed phase and pure quark matter. At this point, the adiabatic index becomes large again and the collapse halts. A shock wave is then produced. One feature of this mechanism is that it requires a particularly soft EoS, since the formation of a mixed phase of quarks and hadrons has to take place at the relatively low densities reached at the moment of core bounce, or immediately after, during the fallback but anyway before deleptonization 64]. Since the densities reached at the moment of the bounce are only moderately dependent on the mass of the progenitor, this mechanism is rather "universal", affecting most of the SNe, although its effect on the explosion can still depend on the mass of the progenitor. This first possibility, QDBD, is not compatible with the two-families scenario, because it would imply that almost all compact stars are quark stars, since quark deconfinement would take place at very low densities.

The second possibility, QDAD, is that quark deconfinement takes places only after an at least partial deleptonization 65 66. It is well known, in fact, that when the pressure due to leptons decreases, the central baryonic density increases and therefore the deconfinement process becomes easier.

The process of deconfinement can then take place in two possible ways: either as a smooth transition or as a first order one, associated with the formation of some intermediate meta-stable phase. In order for the transition to be completely smooth two conditions need to be satisfied: finite-volume effects are irrelevant (so that even a single nucleon can melt into quarks above a given critical density) and no critical value for the strangeness fraction of the quark phase should exist. The latter point is particularly important, because the existence of a minimum critical value for the strangeness fraction implies the existence of a second minimum (either local or global) in the energy per baryon vs density function, separated from the minimum at strangeness equal zero by a barrier. In the case of hybrid stars it is possible to satisfy in particular the second condition, as analyzed e.g. in Ref. 65. Instead, since the two-families scenario is based on the existence of quark stars, a critical value for the strangeness must exist (instead ordinary matter would just decay into strange quark matter). Therefore the process of deconfinement in the two-families scenario always goes through the formation of a metastable phase.

If a critical strangeness fraction needs to be reached in order to deconfine, the question concerns the way one reaches that critical value soon after the pre-supernova collapse. The most simple way is to imagine that due to mass fallback and/or to the slow-down in case of a rapidly rotating star the central density increases. At a certain density hyperons will start being produced. While it is difficult to estimate which can be the critical density of hyperons (and therefore of strangeness) necessary to trigger quark deconfinement, it is clear that it must be at least of the order of a few percent of the total baryons, instead the strange quarks in the hyperons will be too far away one from the other in order to interact and to drive the process of deconfinement. As mentioned in the introduction, it is well possible that statistical fluctuations will play the crucial role, so that at a certain moment a large enough number of hyperons will be contained in a small volume and the process of deconfinement will start.

A important point can be noticed from Fig. 4 of paper 1 : at the temperature reached in the compact star immediately after the collapse, the densities of hyperons are still not very different from the ones at zero temperature. Therefore one can conclude that only the stars having a mass close to about $1.5 M_{\odot}$ will undergo a phase transition soon after the SN explosion. Stars having a mass of about 1.3-1.4 $M_{\odot}$ will not be affected and the mechanism by which they explode will not be linked to quark deconfinement.

It is well known that at the moment the standard mechanism has difficulties in explaining SNae associated with the collapse of massive progenitors, the ones which in principle will generate the most massive neutron stars. A possible way-out is the following: if the proto-neutron star is rapidly rotating (a condition similar to the one needed to produce GRBs in the proto-magnetar model) the rate of fallback can be reduced, allowing the system the time to deconfine and to generate a powerful burst of neutrinos associated with the cooling from the heat released by deconfinement. Notice that the neutrinos are generated at a depth of few $\mathrm{km}$ inside the star and therefore they need a few tenths of a second to start flowing out of the star. 
If the star is rapidly rotating, so that it does not collapse to a black hole during that time, the neutrino flux can be sufficient to revitalize the supernova explosion. In Fig 5 we show the neutrino luminosities computed in Ref. 25] by using a slightly different set of EoS respect to the ones discussed here: the luminosities peak at about $3 \times 10^{52}$ erg, a value comparable to the one obtained at the moment of the collapse. The luminosities computed with the EoSs described in paper 1 would have even higher peaks since the total energy released by deconfinement is larger.

\subsection{SN1987a and fizzlers}

The only SN neutrinos detected till now are those from SN1987A. Indeed, on February 23, 1987, at 2 h 53 m (UT) LSD detector observed 5 events [67]; at $7 \mathrm{~h} 36 \mathrm{~m}$ (UT) IMB, Kamiokande-II and Baksan 68, 69,70 detectors observed 8, 11 and 5 events respectively. The progenitor was a blue supergiant with estimated mass of $\sim 20 M_{\odot}$.

The observations of Kamiokande-II, IMB and Baksan can be explained very well within the standard scenario for core collapse SNe, assuming that the events are due to $\bar{\nu}_{e} p \rightarrow n e^{+}$. The observations are consistent with the presence of an initial, high luminosity phase of neutrino emission, followed by a thermal phase due to the cooling of the newborn neutron star [71,72]. Such an initial and luminous phase is expected; indeed, it should trigger the subsequent explosion of the star. The standard scenario for core collapse $\mathrm{SNe}$ does not predict the existence of multiple pulses of neutrino emission and thus cannot accommodate LSD data.

An interesting possibility is that the first burst is due to a very intense neutronization phase by $e^{-} p \rightarrow n \nu_{e}$; it was noted in $[73$ that electron neutrinos with an energy of $30-40 \mathrm{MeV}$ can be more easily seen in the LSD detector than in the other detectors. In the astrophysical scenario of 73 , the rapid rotation of the collapsing core leads to a delay between the first and the second burst. However, the nature of the second burst is not discussed in 73 .

In Ref. 63] it was discussed the possibility that KamiokandeII, Baksan and IMB observations are due to the burning of hadrons into quarks. The sequence of events, in that case, could be the following:

- the rapid rotation of the collapsing core halts the collapse at subnuclear densities, forming a so-called "fizzler" 74];

- an initial intense phase of neutronization accounts for the LSD observations as in 73;

- the rapid rotation of the core leads to the formation of a metastable neutron star, that looses its angular momentum in a time scale of several hours;

- the central density of the metastable star becomes large enough that deconfinement can take place. Again, the rapid release of energy at the beginning of the last stage could be sufficient to lead to the explosion of the star.

This scheme, although interesting, is strictly based on the possibility of having very large values of angular momentum in the central region of the star, what seems at odd with the results of Ref. [75. On the other hand a similar criticism can be applied to the model for GRBs based on the formation of a millisecond proto-magnetar, a model that is having a great phenomenological success. We think therefore that the analysis of the distribution of the angular momentum in the collapsing core cannot yet be considered concluded.

\section{Comparison with other models}

The possible connection between quark deconfinement and explosive astrophysical phenomena has a relatively long story. The papers discussing this relation have concentrated on some specific associations. The oldest proposed connections have to do with suggestions on how deconfinement can help SNae to explode by providing a soft EoS in the mixed phase, followed by a stiff EoS in the pure quark matter phase 61,62, on how GRBs can be associated with the energy released by the deconfinement [46. [76. and on how quark stars can help to generate the GRB by providing a cleaner environment [77,78.

The discovery of very massive compact stars has changed the scenario concerning the possible impact of quark deconfinement on SNae and on GRBs, since one needs to clarify the composition of the most massive stars before discussing the transition from hadronic to hybrid or quark stars. As we have tried to clarify in this review, once a specific proposal for the EoS of matter at high densities has been formulated, the possible transitions from hadronic to quark (or, in other schemes, hybrid) stars appear in a natural way and the phenomenological implications can be outlined rather precisely.

An attempt at systematizing a variety of phenomena into a unique scheme has been made during many years by Ouyed and collaborators. The scheme they have developed, named Quark-Nova, is based on the idea that the process of deconfinement takes place as a detonation and that therefore quite a significant amount of matter is ejected by a mechanical shock at the end of the process. The mass ejection can interact with the material already present in the surroundings of the compact star and it can originate a variety of phenomena: nucleosynthesis in neutron-rich ejecta [79]; GRBs, both by releasing a huge amount of energy from the surface of the quark star via photon emission 80 and also by using the interaction of the ejecta from the Quark-Nova with the ejecta of the preceding SN in order to generate a late-time x-ray emission [81. Also it has been proposed to explain the long-time duration and the spectral features of SN 2006gy as due to the interaction of the Quark-Nova ejecta with the ejecta of the preceding SN 82 .

While the suggested associations between explosive phenomena and quark deconfinement are very interesting, two inter-correlated questions arise. First, explicit analysis of the process of quark deconfinement are not indicating a detonation, but a deflagration, as discussed at the beginning of this review. Second, it would be interesting to see if the detonation plays really the crucial role or if the mass 
ejected e.g. by neutrino ablation in the case of a deflagration can be sufficient to interpret some of the phenomena as due to quark's deconfinement but not to the specific Quark-Nova model. A work in that direction is e.g. 83, indicating a significant amount of mass ejection during the formation of a quark star due to the neutrino emission. This result is compatible with the more recent analysis we made 10 and it opens the possibility of re-discussing some of the phenomena by using a deflagration instead of a detonation.

\section{Conclusions}

In this and in the accompanying paper 1 we have presented the two-families scenario and we have discussed the many implications it has on astrophysics. We have seen in paper 1 that the measure of the radius of a few compact stars would likely confirm or rule-out the model. Possible confirmations could also come from the study of LMXBs which are displaying in a few cases large eccentricity whose origin is still unknown, and it could be originated by the phase transition to quark star of the neutron star in the binary system.

The most spectacular implications of the two-families scenario are though probably connected with explosive phenomena and in particular with short GRBs. First, a direct outcome of the two-families scenario is that if a compact star and not a $\mathrm{BH}$ forms after the merging than that object is a quark star. This very strong implication can be tested e.g. by studying gravitational waves emitted immediately before and immediately after the merger (see e.g. the review paper by Bauswein, Stergioulas and Janka). Another striking implication of the formation of a quark star immediately after the merger is the possibility of explaining both long and short GRBs by using the proto-magnetar model as described in Sec4.1. Notice that in these two examples one would not generically test the formation of quark matter inside the compact star, but the formation of a quark star, and therefore the two-families scenario.

While many aspects of the scenario still need to be worked out, as for instance the behaviour of the magnetic field during the formation of the quark star, we are confident that in the near future the scenario will be tested and therefore confirmed or ruled out by a multitude of experiments and observations, ranging from the analysis of GW emission, to the measure of the radii of compact stars, to the analysis of the emission of GRBs. The possibility of being tested is ultimately the divide between a theory and a speculation.

A.D. would like to thank V. Hislop for the moral support during the preparation of the paper. G.P. acknowledges financial support from the Italian Ministry of Research through the program Rita Levi Montalcini.

\section{References}

1. A. Drago, A. Lavagno, G. Pagliara, Phys.Rev. D89, 043014 (2014).

2. M. Bejger, J.L. Zdunik, P. Haensel, M. Fortin, Astron. Astrophys. 536, A92 (2011).

3. Y. Sekiguchi, K. Kiuchi, K. Kyutoku, M. Shibata, Phys. Rev. Lett. 107, 211101 (2011).

4. A. Bodmer, Phys.Rev. D4, 1601 (1971).

5. E. Witten, Phys. Rev. D30, 272 (1984).

6. K. Iida, K. Sato, Phys.Rev. C58, 2538 (1998).

7. I. Bombaci, D. Logoteta, P. Panda, C. Providencia, I. Vidana, Phys.Lett. B680, 448 (2009).

8. B. Mintz, E. Fraga, G. Pagliara, J. Schaffner-Bielich, Phys.Rev. D81, 123012 (2010).

9. I. Bombaci, D. Logoteta, C. Providencia, I. Vidana, Astron. Astrophys. 528, A71 (2011).

10. A. Drago and G. Pagliara, Phys. Rev. C 92 (2015) 4, 045801.

11. C. Alcock, E. Farhi, A. Olinto, Astrophys.J. 310, 261 (1986).

12. P. Haensel, J. Zdunik, R. Schaeffer, Astron.Astrophys. 160, 121 (1986).

13. A.V. Olinto, Physics Letters B 192, 71 (1987).

14. B. Niebergal, R. Ouyed, P. Jaikumar, Phys.Rev. C82, 062801 (2010).

15. M.G. Alford, S. Han, K. Schwenzer, Phys.Rev. C91, 055804 (2015).

16. L.D. Landau, E.M. Lifshitz, Fluid Mechanics, Second Edition: Volume 6 (Course of Theoretical Physics), Course of theoretical physics / by L. D. Landau and E. M. Lifshitz, Vol. 6, 2nd edn. (Butterworth-Heinemann, 1987), ISBN 0750627670

17. W. Hillebrandt, J.C. Niemeyer, Ann. Rev. Astron. Astrophys. 38, 191 (2000).

18. S.I. Blinnikov, P.V. Sasorov, S.E. Woosley, Space Science Reviews 74, 299 (1995).

19. F. Williams, Combustion theory (The Benjamin/Cummings Publishing Company, Inc., Menlo Park, California (USA), 1985).

20. B. Coll, Annales de l'I.H.P. A 25, 363 (1976).

21. A. Anile, Relativistic Fluids and Magneto-fluids (Cambridge University Press, 1990).

22. A. Drago, G. Pagliara (2015), 1504.02795.

23. A. Drago, A. Lavagno, I. Parenti, Astrophys.J. 659, 1519 (2007).

24. M. Herzog, F.K. Röpke, Phys.Rev. D84, 083002 (2011).

25. G. Pagliara, M. Herzog, F. Ropke, Phys.Rev., D87, 103007 (2013).

26. S. Shapiro, S. Teukolsky, Black holes, white dwarfs, and neutron stars: The physics of compact objects (Wiley \& Sons, New York, Chichester Brisbane, Toronto, Singapore, 1983).

27. P. Meszaros, Rept. Prog. Phys. 69, 2259 (2006).

28. E. Berger, Ann. Rev. Astron. Astrophys. 52, 43 (2014).

29. A. MacFadyen, S.E. Woosley, Astrophys. J. 524, 262 (1999).

30. A.I. MacFadyen, S.E. Woosley, A. Heger, Astrophys. J. 550, 410 (2001).

31. B.D. Metzger, D. Giannios, T.A. Thompson, N. Bucciantini, E. Quataert, Mon. Not. Roy. Astron. Soc. 413, 2031 (2011). 
32. P.A. Mazzali, A.I. McFadyen, S.E. Woosley, E. Pian, M. Tanaka, Mon. Not. Roy. Astron. Soc. 443, 67 (2014).

33. J.S. Bloom, S.R. Kulkarni, S.G. Djorgovski, Astron. J. 123, 1111 (2002).

34. A. Heger, C.L. Fryer, S.E. Woosley, N. Langer, D.H. Hartmann, Astrophys. J. 591, 288 (2003).

35. J. Sollerman et al., Astron. Astrophys. 454, 503 (2006).

36. P.A. Mazzali, J.S. Deng, N. Tominaga, K. Maeda, K. Nomoto, T. Matheson, K.S. Kawabata, K.Z. Stanek, P.M. Garnavich, Astrophys. J. 599, L95 (2003).

37. S.R. Kulkarni, D.A. Frail, M.H. Wieringa, R.D. Ekers, E.M. Sadler, R.M. Wark, J.L. Higdon, E.A. Phinney, Nature 395, 663 (1998).

38. J.P.U. Fynbo et al., Nature 444, 1047 (2006).

39. A. Drago, G. Pagliara, Astrophys.J. 665, 1227 (2007).

40. E. Nakar, T. Piran, Mon. Not. Roy. Astron. Soc. 331, 40 (2002).

41. C. Guidorzi, S. Dichiara, F. Frontera, R. Margutti, A. Baldeschi, L. Amati, Astrophys. J. 801, 57 (2015).

42. N. Lyons, P. O'Brien, B. Zhang, R. Willingale, E. Troja et al., Mon.Not.Roy.Astron.Soc. 402, 705 (2010).

43. S. Dall'Osso, G. Stratta, D. Guetta, S. Covino, G. De Cesare et al., Astron.Astrophys. 526, A121 (2011).

44. B.B. Zhang, D.N. Burrows, B. Zhang, P. Meszaros, G. Stratta et al., Astrophys.J. 748, 132 (2012).

45. D. Gruber et al., Astron. Astrophys. 528, A15 (2011).

46. Z.G. Dai, T. Lu, Phys. Rev. Lett. 81, 4301 (1998).

47. M.A. Ruderman, L. Tao, W. Kluzniak, Astrophys. J. 542, 243 (2000).

48. J. Heyvaerts, S. Bonazzola, M. Bejger, P. Haensel, Astron. Astrophys. 496, 317 (2009).

49. M. Della Valle et al., Nature 444, 1050 (2006).

50. A. Gal-Yam et al., Nature 444, 1053 (2006).

51. T. Moriya, N. Tominaga, M. Tanaka, K. Nomoto, D. N. Sauer, P. A. Mazzali, K. Maeda and T. Suzuki, Astrophys. J. 719 (2010) 1445.

52. V. Paschalidis, Y.T. Liu, Z. Etienne, S.L. Shapiro, Phys. Rev. D84, 104032 (2011).

53. D. Lazzati, E. Ramirez-Ruiz, G. Ghisellini, Astron. Astrophys. 379, L39 (2001).

54. Y. Kaneko, R.D. Preece, M.S. Briggs, W.S. Paciesas, C.A. Meegan, D.L. Band, AIP Conf. Proc. 836, 133 (2006), [Astrophys. J. Suppl.166,298(2006)].

55. G. Ghirlanda, L. Nava, G. Ghisellini, A. Celotti, C. Firmani, Astron. Astrophys. 496, 585 (2009).

56. G. Calderone et al., Mon. Not. Roy. Astron. Soc. 448, 403 (2015).

57. A. Rowlinson, P.T. O'Brien, B.D. Metzger, N.R. Tanvir, A.J. Levan, Mon. Not. Roy. Astron. Soc. 430, 1061 (2013).

58. H.J. Lu, B. Zhang, W.H. Lei, Y. Li, P.D. Lasky, Astrophys.J. 805, 89 (2015).

59. B. Paczynski, P. Haensel, Mon. Not. Roy. Astron. Soc. 362, L4 (2005).

60. A. Drago, A. Lavagno, B. Metzger, G. Pagliara, arXiv:1510.05581 [astro-ph.HE].

61. N. Gentile, M. Aufderheide, G. Mathews, F. Swesty, G. Fuller, Astrophys.J. 414, 701 (1993).

62. A. Drago, U. Tambini, J.Phys. G25, 971 (1999).

63. A. Drago, G. Pagliara, G. Pagliaroli, F.L. Villante, F. Vissani, AIP Conf.Proc. 1056, 256 (2008).

64. I. Sagert, T. Fischer, M. Hempel, G. Pagliara, J. SchaffnerBielich et al., Phys.Rev.Lett. 102, 081101 (2009).
65. J.A. Pons, A.W. Steiner, M. Prakash, J.M. Lattimer, Phys.Rev.Lett. 86, 5223 (2001).

66. D.N. Aguilera, D. Blaschke, H. Grigorian, Astron. Astrophys. 416, 991 (2004).

67. M. Aglietta et al., Europhys. Lett. 3, 1315 (1987).

68. R.M. Bionta et al., Phys. Rev. Lett. 58, 1494 (1987).

69. K. Hirata et al. (Kamiokande-II), Phys. Rev. Lett. 58, 1490 (1987).

70. E.N. Alekseev, L.N. Alekseeva, I.V. Krivosheina, V.I. Volchenko, Phys. Lett. B205, 209 (1988).

71. T.J. Loredo, D.Q. Lamb, Phys. Rev. D65, 063002 (2002).

72. G. Pagliaroli, F. Vissani, M.L. Costantini, A. Ianni, Astropart. Phys. 31, 163 (2009).

73. V.S. Imshennik, O.G. Ryazhskaya, Astron. Lett. 30, 14 (2004).

74. J.N. Imamura, B.K. Pickett, R.H. Durisen, Astrophys. J. 587, 341 (2003).

75. A. Heger, S.E. Woosley, H.C. Spruit, Astrophys. J. 626, 350 (2005).

76. I. Bombaci, B. Datta, Astrophys.J. 530, L69 (2000).

77. B. Paczynski, Astrophys. J. 308, L43 (1986).

78. P. Haensel, B. B. Paczynski, P. P. Amsterdamski, Astrophys. J. 375, 209 (1991).

79. P. Jaikumar, B.S. Meyer, K. Otsuki, R. Ouyed, Astron. Astrophys. 471, 227 (2007).

80. R. Ouyed, F. Sannino, Astron.Astrophys. 387, 725 (2002).

81. R. Ouyed, J. Dey, M. Dey, Astron. Astrophys. 390, L39 (2002).

82. R. Ouyed, M. Kostka, N. Koning, D. Leahy, W. Steffen, Mon. Not. Roy. Astron. Soc. 423, 1652 (2012).

83. P. Keranen, R. Ouyed, P. Jaikumar, Astrophys.J. 618, 485 (2004). 
